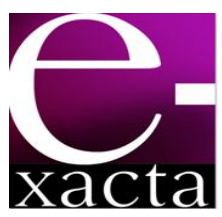

ISSN: 1984-3151

\section{ATIVIDADE INTEGRALIZADORA: O ENSINO DE ENGENHARIA SOB UMA NOVA PERSPECTIVA}

\author{
INTEGRATED ACTIVITY: THE TEACHING OF ENGINEERING IN A NEW \\ PERSPECTIVE
}

\author{
Bruno Edward Meyer'; Denilton da Conceição Fritz Junior²; Elis Regina Duarte³; Maria \\ Cecilia Meneguette Ferreira ${ }^{4}$; Paula Cristiana de Freitas ${ }^{5}$
}

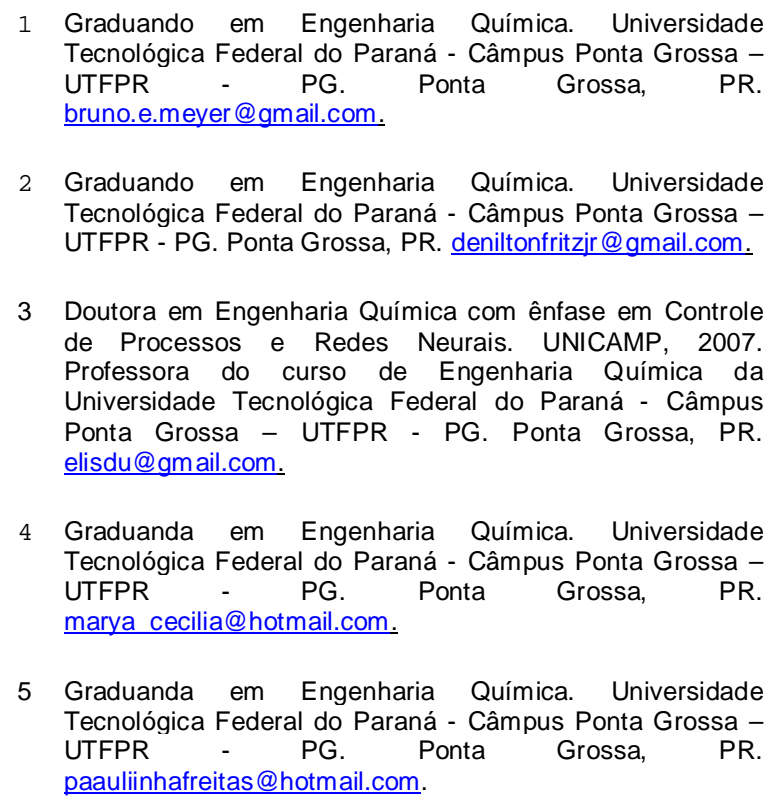

Recebido em: 20/04/2014 - Aprovado em: 30/04/2014 - Disponibilizado em: 31/05/2014

RESUMO: Ao se observar as barreiras encontradas por acadêmicos de períodos distintos durante a graduação em Engenharia Química da Universidade Tecnológica Federal do Paraná - Campus Ponta Grossa (UTFPR - PG), constatou-se que as principais dificuldades envolviam o correlacionamento das matérias básicas da área de Engenharia e o reconhecimento acerca da importância do aprendizado envolvido nessas disciplinas. Inserido na função de auxiliar na formação interdisciplinar do acadêmico a fim de buscar soluções para as problemáticas em questão, o Programa de Educação Tutorial do curso de Engenharia Química (PET EQ) da UTFPR - PG, caracterizado por ações implementadas junto às graduações das Instituições de Ensino Superior, desenvolveu a Atividade Integralizadora. A atividade buscou facilitar o processo de aprendizagem do acadêmico e permitir a ele a visualização das áreas de atuação do profissional Engenheiro. A realização de uma abordagem lúdica e dinâmica instigou os acadêmicos a provar o conhecimento adquirido nas salas de aula e provocou abertura a novos conceitos de ensino.

PALAVRAS-CHAVE: Ensino. Integralizadora. Indissociabilidade. Engenharia Química. Ciclone.

ABSTRACT: By observing the barriers faced by academics from different periods during graduation in Chemical Engineering from the Federal Technological University of Paraná - Campus Ponta Grossa (UTFPR - PG), it was found that the main difficulties involved correlating the core subject area of Engineering and recognition of the importance of learning involved in these disciplines. Inserted in the auxiliary function in interdisciplinary academic and seek solutions to the problem in question, the Tutorial Education Program of the course of Chemical Engineering (PET EQ) from UTFPR - PG, actions implemented with the graduations of Institutions of Higher Education, developed the Integrated Activity. The activity aimed to facilitate the learning process of academic and 
allow him to visualize the areas of the professional Engineer. Conducting a playful and dynamic approach urged academics to prove the knowledge acquired in the classroom and caused the opening to new concepts of teaching. KEYWORDS: Education. Integrated. Inseparability. Chemical Engineering. Cyclone.

\section{INTRODUÇÃO}

A situação atual de desenvolvimento do mercado global mais competitivo necessita, cada vez mais, de profissionais aptos para a continuidade da construção da sociedade. Todas as transformações, ocorridas nos últimos anos, exigem também mudanças no que diz respeito principalmente à formação dos profissionais ligados às áreas de tecnologias e engenharias.

O desenvolvimento acelerado das indústrias não só faz com que o mercado de trabalho cada vez mais exija dos profissionais qualificação e eficiência, mas requer uma retomada das relações entre esse cenário e o que está posto como modelo de ensino e formação dos engenheiros (SILVA; CECÍLIO, 2007).

No que diz respeito a ensino-aprendizado, é imprescindível que sejam criadas novas abordagens e desenvolvidas ferramentas dinâmicas que proporcionem a construção do saber de maneira mais sólida e abrangente, a fim de também tornar o aprendizado mais atraente para os envolvidos. Segundo Rieder, Zanelatto e Brancher (2004): "O interesse em aprender é despertado no aluno através de ambientes lúdicos que harmonizam conhecimento e prática dos conceitos repassados em sala de aula". São profundas as problemáticas visualizadas nas graduações das áreas de engenharia, tendo em vista que a quase totalidade das disciplinas de conhecimentos específicos exigem a consolidação de um grande número de matérias de conhecimentos básicos, o que distancia o acadêmico da atuação profissional, principalmente em seus primeiros anos de formação.

A problemática do insucesso e evasão de acadêmicos de áreas da Engenharia deve ser confrontada com a intervenção de práticas que visem 0 estímulo à utilização de recursos e metodologias centradas em modelos de tutorias individuais e trabalhos em grupo, onde os alunos possam ter espaço de autonomia, de exploração, mas simultaneamente de apoio face às dificuldades sentidas, respeitando e respondendo às naturais diferenças interindividuais dos alunos de ensino superior (VASCONCELOS; ALMEIDA; MONTEIRO, 2009).

Em virtude da necessidade observada de se reformular a abordagem educacional utilizada nos níveis de graduação, revela-se a importância de se abrir novas possibilidades, frente aos modelos tradicionais adotados nos espaços das salas de aula na transmissão de conhecimentos. Assim, inserido nesse contexto, o Programa de Educação Tutorial (PET) surge com o intuito de desenvolver estratégias que sejam capazes de suprir tais necessidades. $O$ PET é um programa do Governo Federal financiado pelo Ministério da Educação, que se caracteriza pelo desenvolvimento de ações promovidas junto às graduações das Universidades públicas brasileiras, cuja estrutura é integrada por acadêmicos (petianos) que são acompanhados por um professor ligado à graduação (tutor). Tal programa, tem por concepção, o desenvolvimento de ações que valorizem a indissociabilidade da tríade ensino, pesquisa e extensão, de modo a promover atividades de cunho integralizador e visar o desenvolvimento de profissionais completos, com senso crítico e que reconheçam a sua atuação efetiva na sociedade como agentes transformadores. Por isso, o Grupo PET de Engenharia Química (PET EQ) da Universidade Tecnológica Federal do Paraná - Campus Ponta Grossa (UTFPR - PG) trabalha em conjunto com o colegiado do curso e tem a finalidade de permitir aos alunos da graduação, seu envolvimento em projetos 
integradores, articulados entre teoria e prática e nas diferentes áreas do conhecimento.

Nesse sentido, ao se visualizar as dificuldades de aprendizagem enfrentadas pelos acadêmicos do curso de Engenharia Química da UTFPR - PG, frente à necessidade de formular novas metodologias de ensino nas áreas de engenharias, o Grupo PET EQ, na sua função de buscar uma formação interdisciplinar e diferenciada do acadêmico, desenvolveu uma atividade a fim de possibilitar a contextualização do aluno no cotidiano do profissional Engenheiro, e apresentar as disciplinas ministradas em salas de aulas de maneira articulada e integrada.

Diante dessa realidade, indagou-se: De que maneira deve-se reformular a abordagem educacional utilizada nos níveis de graduação? Ao incorporar esse propósito à realidade do ensino superior em engenharia, como é possível comprovar a relação da teoria e prática como princípios não dicotômicos, e alvitrar uma abordagem que facilite o processo de aprendizagem do acadêmico? Aliado a isso, é possível demonstrar as áreas de atuação do Engenheiro?

A fim de buscar soluções para tais questionamentos, foi proposta a elaboração da Atividade Integralizadora. Tal atividade consistiu na realização de um exercício prático que envolveu o uso das disciplinas lecionadas no primeiro período do curso. Para isso, os conceitos, adquiridos de forma isolada nas salas de aulas, foram expostos correlacionados e interdisciplinarmente mediante a apresentação de um processo real da Engenharia Química, a centrifugação. Assim, para permitir a visualização da importância de cada disciplina e a vivência envolvida na utilização delas, os acadêmicos utilizaram ferramentas de gestão e foram instigados a comprovar seu conhecimento, desenvolvendo um projeto de um equipamento industrial, aplicado no processo de centrifugação, o ciclone.
Dessa forma, o presente artigo constitui, na próxima seção, uma revisão bibliográfica sobre centrifugação. Em seguida, expõe-se a metodologia aplicada no desenvolvimento da atividade e realiza-se uma avaliação e discussão sobre os resultados obtidos. Por fim, a última seção apresenta uma conclusão do trabalho.

\section{REVISÃo BIBLIOGRÁFICA}

Seja o conceito de centrifugação dado a seguir.

A centrifugação é um processo físico-mecânico que tem por função a separação ou clarificação de uma mistura sólido-fluido, em que as partículas, de tamanhos e densidades diferentes, são separadas do fluido pela ação de uma força centrípeta. (GEANKOPLIS, 1993)

Para a realização de tal operação, são utilizadas centrífugas, cujo uso proporciona um aumento considerável das forças que atuam sobre as partículas.

Algumas vezes a separação pode ser realizada por gravidade, mas é demasiada lenta, devido à proximidade das densidades da partícula e do fluido ou devido às forças de associação que mantém unidos os componentes, como no caso das emulsões. A separação por centrifugação é utilizada em muitas indústrias alimentícias, tais como cervejarias, processamento de óleos vegetais, concentração de proteína de pescado, processamento de sumos de frutas suco para remoção de material celular, secagem de cristais, separação de constituintes líquidos e sólidos das emulsões, entre outros (GEANKOPLIS, 1993).

Desta forma, um dos equipamentos mais aplicados na indústria de particulados são os ciclones.

Os ciclones são amplamente utilizados como separador gás-sólido, pois sua estrutura é simples e possui baixo custo de operação, e podem ser adaptados em operações de condições extremas como: altas pressões, altas temperaturas e gases corrosivos, desde que usado o material apropriado na sua construção. (...) O ciclone é composto por uma parte cônica e outra cilíndrica, que juntas formam o corpo do equipamento. A sua entrada é usualmente tangencial à parede da parte cilíndrica próxima ao topo. O tubo de saída do gás, usualmente chamado de vórtex, ou finder, é fixo na parte superior do ciclone. Os separadores ciclônicos são amplamente utilizados para remoção de partículas do ar ou de gases de processos. São utilizados também como reator químico, trocador de 
calor, para secagem de materiais granulares e combustão de óleo (SILVA, 2006).

No ciclone, o gás carregado de pó entra no sentido tangencial pela câmara cilíndrica ou cônica a uma alta velocidade, por uma ou mais de uma abertura e sai por um orifício central. Dentro do ciclone, as partículas são submetidas, na direção radial, a dois tipos de forças opostas: a força centrífuga e a de arraste. A força centrífuga tende a empurrar partículas para as paredes do ciclone, ao passo que a força de arraste atua no sentido de arrastar as partículas junto com o gás na saída do ciclone. Essas forças são função do raio de rotação e do tamanho das partículas; partículas com tamanhos diferentes giram em raios diferentes. A Figura 1 apresenta as vistas de um ciclone e o movimento das partículas e do gás dentro do equipamento.

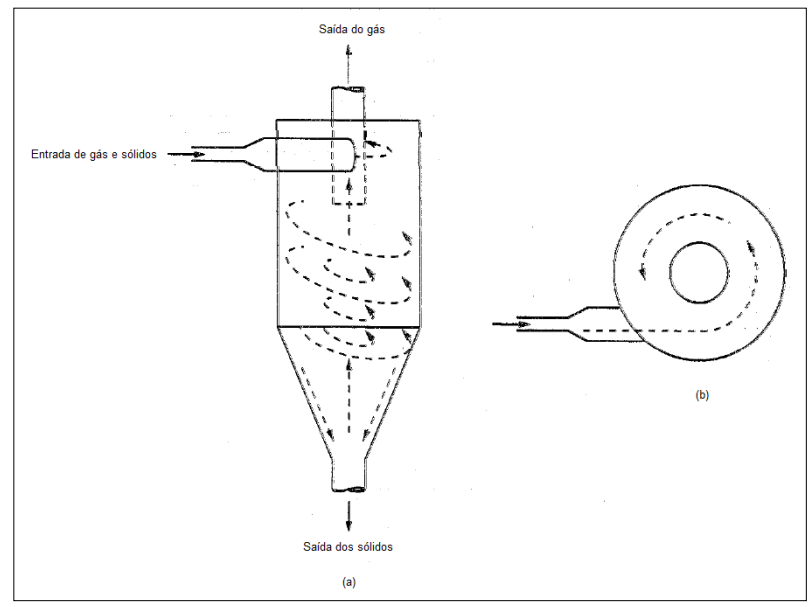

Figura 1 - Esquema de uma separação gás-sólido de um ciclone: a) vista lateral, b) vista superior

Fonte: GEANKOPLIS, 1993, p. 928.

\section{Metodologia}

Os conceitos mais aprofundados, que envolvem o entendimento sobre o processo de funcionamento dos ciclones, o projeto do equipamento e as adequações de sua utilização, são abordados em disciplinas mais avançadas da graduação em Engenharia Química, pois tais fundamentações específicas necessitam de conhecimentos previamente trabalhados nos períodos iniciais. Sendo assim, durante a realização da Atividade Integralizadora, os conceitos que envolviam o desenvolvimento do projeto de um equipamento da indústria química foram abordados, para os acadêmicos do primeiro período, de forma mais genérica. Com isso, buscou-se utilizar fundamentos já trabalhados pelos alunos nas disciplinas daquele período. Para execução da atividade, foram constituídos quatro grupos, com a proposta de ampliar a interação dos participantes e também avaliar com maior precisão o desenvolvimento de todos os envolvidos.

$\mathrm{Na}$ realização da primeira etapa da Atividade Integralizadora, aplicou-se a ferramenta Quiz. Essa ferramenta pode ser definida como um conjunto de perguntas concisas que precisam ser respondidas pelos participantes em um determinado tempo, como uma prova ou um teste objetivo.

Sua utilização propõe facilitar o aprendizado do acadêmico e tornar o ensino mais dinâmico, podendo até aumentar o interesse pela temática, auxiliando, dessa forma, o processo de ensinoaprendizagem. (SILVA et al., 2010).

Assim, o Quiz da Atividade Integralizadora contemplou vinte questões e envolveu conceitos trabalhados nas disciplinas de Física, Química e Operações Unitárias, aplicados na temática de separação sólido-fluido. Cada questão respondida corretamente pelo grupo participante configurava uma pontuação que, ao final do processo, permitia o acesso a recursos (materiais didáticos) que seriam utilizados para solucionar problemáticas da etapa subsequente.

Em seguida, a próxima etapa da atividade consistia em simular, com os acadêmicos, o desenvolvimento de um negócio na área de projetos de Engenharia, a qual, no caso, abrangia o projeto, construção e venda de ciclones industriais. Assim, cada grupo recebeu um Cartão de Projetos e um Cartão de Dicas. Esses cartões possuíam variáveis específicas, que compreendiam a descrição, as equações e dados do 
projeto, utilizados no ciclone requerido. Para possibilitar a construção do equipamento, além dos recursos previamente adquiridos durante a primeira etapa, as vistas do ciclone foram disponibilizadas no projetor da sala. Por fim, a ferramente "Business Model Canvas" (BMC), ou "Painel de Modelo de
Negócios", foi utilizada no intuito de facilitar o processo de negociação do ciclone e entendimento acerca das problemáticas envolvidas no projeto.

O modelo de Cartão de Projeto, utilizado no desenvolvimento da atividade, pode ser visualizado na Figura 2.

\section{CARTÃO DE PROJETO NO3}

Projeteum ciclonepara a indústria de mineração, atentando para as especificaçães da mesma.

- O ciclone deve ser construido em material não poroso.

- A mistura contém particulas de galena com densidade de $7500 \mathrm{~kg} / \mathrm{m}^{3} \mathrm{e}$ diâmetro de corte de $0,0004872 \mathrm{~m}$.

- A velocidade do fluido que entra no ciclone é de $7 \mathrm{~m} / \mathrm{s}$.

- O fluido utilizado é a água.

- O ciclone deve ser um ciclone de Lapple com diâmetro duas vezes maior que a largura do duto de entrada.

- A empresa pretende investir na melhoria da qualidade da produção,e minimizar os custos de manutenção.

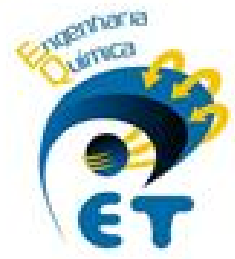

Figura 2 - Modelo de Cartão de Projeto, utilizado no desenvolvimento da atividade

O BMC é uma ferramenta de gestão estratégica e empresarial, que contribui de forma significativa para a construção rápida e visual de novos produtos ou serviços. A aplicação do BMC consiste no uso de um painel dividido em nove grandes blocos, que representam os elementos fundamentais que compõem um modelo de negócio. O objetivo central no exercício do preenchimento do painel é extrair propostas de valor que atendam e potencializam os principais objetivos desejados, antes de partir de fato para a formatação do produto ou serviço. A Figura 3 apresenta o formato da ferramenta de gestão BMC.
Assim, mediante o uso do BMC, os acadêmicos estruturaram o plano de negócios a partir do desenvolvimento do projeto de seus equipamentos. A aplicação da ferramenta de gestão permitiu que os grupos visualizassem 0 contexto envolvido no processo, como exigências dos clientes e matériasprimas necessárias para a construção dos equipamentos.

A utilização dos cartões possibilitou o levantamento das variáveis do projeto e viabilizou a construção de um protótipo do ciclone. Para finalizar a atividade, cada grupo (Figura 4) apresentou aos demais 
participantes o protótipo construído e o modelo de negócios proposto.

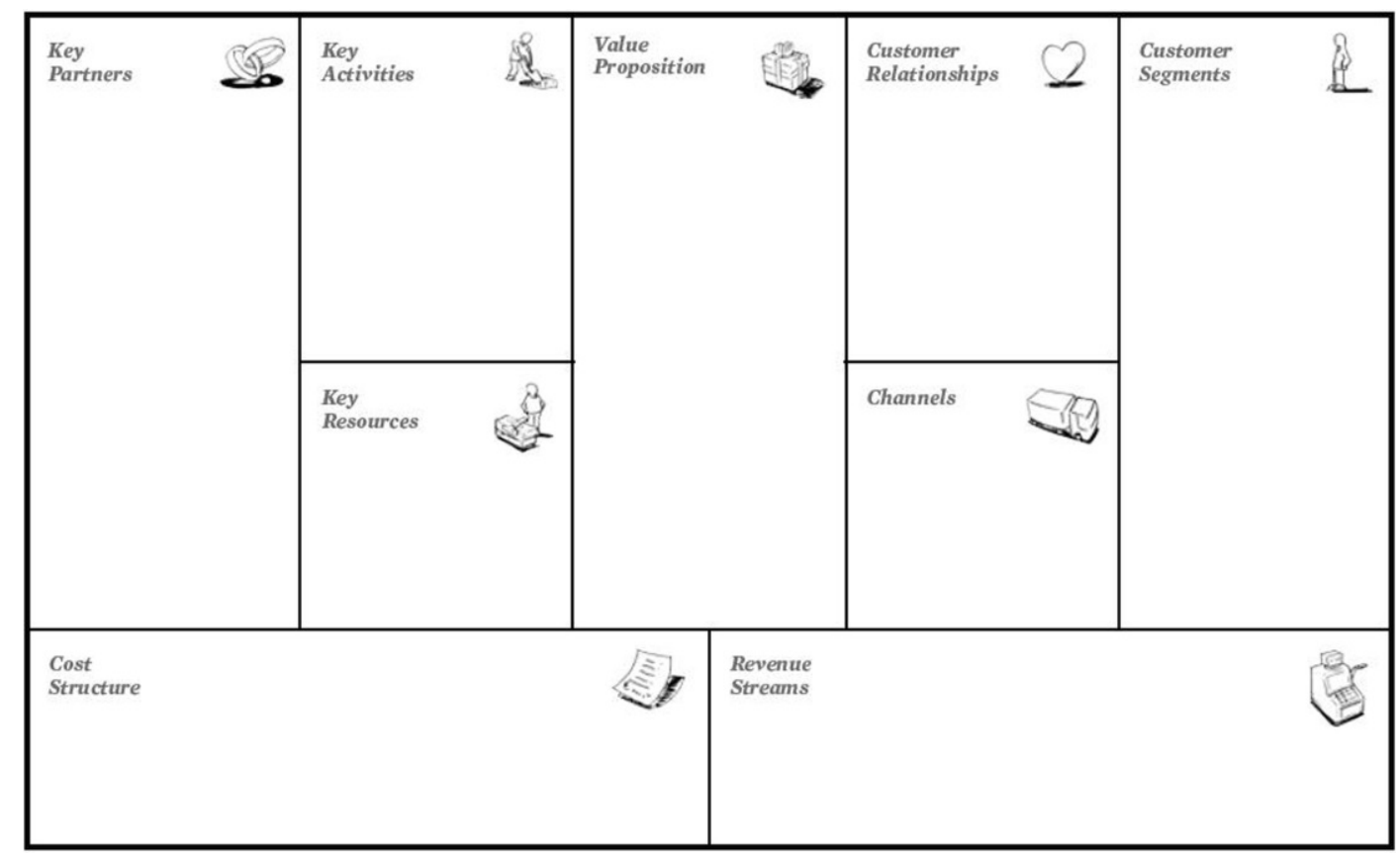

Figura 3 - Formato da ferramenta de gestão BMC

Fonte: Business Model Foundry $\mathrm{GmbH}$.

Disponível em: www.businessmodelgeneration.com

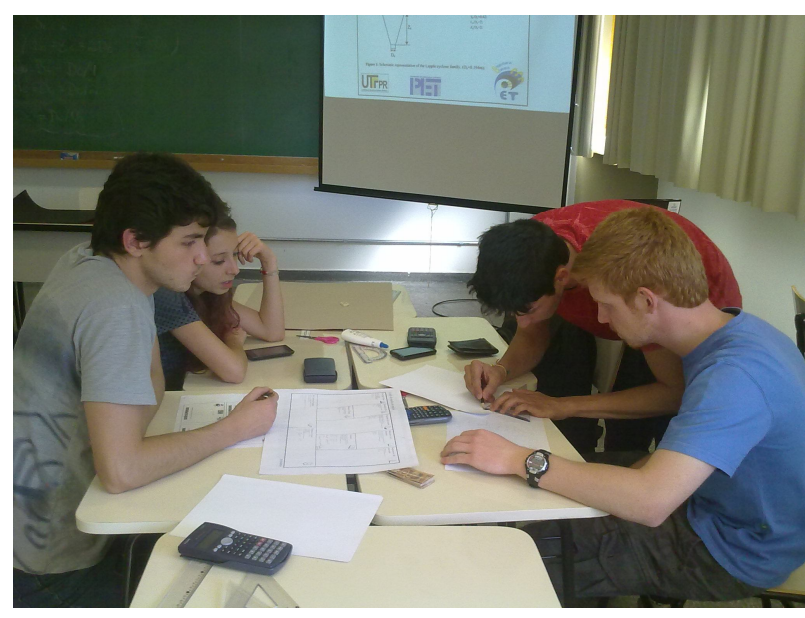

Figura 4 - Grupo participante da atividade, trabalhando no desenvolvimento do projeto

Por fim, cada acadêmico recebeu um Questionário Avaliativo de Atividade (QAA), o qual consistia de um formulário com sete questões subjetivas que abordavam o desenvolvimento do trabalho realizado no dia. Esse formulário foi preenchido pelos participantes e entregue ao Grupo PET EQ no mesmo dia. A Tabela 1 apresenta as perguntas realizadas.

Tabela 1

Perguntas referentes ao "Questionário Avaliativo de Atividade"

\begin{tabular}{c|c}
\hline Número & Perguntas \\
\hline 1 & $\begin{array}{c}\text { Como você avalia a Atividade } \\
\text { Integralizadora? }\end{array}$ \\
\hline 2 & $\begin{array}{c}\text { Você acredita que essa atividade } \\
\text { contribuiu para seu desenvolvimento } \\
\text { pessoal e/ou profissional? }\end{array}$ \\
\hline 3 & $\begin{array}{c}\text { Como você avalia o desenvolvimento da } \\
\text { atividade dentro do seu grupo? }\end{array}$ \\
\hline 4 & $\begin{array}{c}\text { Como você avalia a participação dos } \\
\text { demais acadêmicos na atividade? }\end{array}$ \\
\hline 5 & $\begin{array}{c}\text { Você acredita que mais atividades desse } \\
\text { domínio deveriam ser realizadas com } \\
\text { maior frequência? }\end{array}$ \\
\hline 6 & O que você aprendeu hoje? \\
\hline 7 & $\begin{array}{c}\text { Cite algumas sugestões de temas para as } \\
\text { próximas atividades. }\end{array}$ \\
\hline
\end{tabular}




\section{Discussão dos Resultados}

Inicialmente, a atividade foi apresentada aos acadêmicos, no intuito de facilitar o entendimento sobre os trabalhos que os alunos iriam realizar. Desta forma, percebe-se que foi possível direcioná-los com mais facilidade, tendo em vista que eles já visualizavam as diretrizes da atividade. Em seguida, os participantes se dividiram em grupos e iniciaram o envolvimento nos trabalhos.

Primeiramente, a ferramenta Quiz foi utilizada como estratégia de aumento do interesse dos acadêmicos na busca pelo conhecimento de Engenharia. Esta primeira etapa, que consistia em um conjunto de vinte questões de múltipla escolha, referentes aos processos de separação sólido-fluido, foram bastante úteis, no sentido de contextualizar os conceitos e fundamentos teóricos que seriam utilizados pelos participantes na sequência, para o desenvolvimento no projeto do protótipo do ciclone (Figura 5). Visualizou-se que, algumas questões foram respondidas, pois exigiam do acadêmico apenas um conhecimento prévio, já adquirido, enquanto que outros questionamentos envolviam conceitos e fundamentos teóricos desenvolvidos em períodos subsequentes e, por isso, não puderam ser respondidos. Desta forma, foi possível instigar nos acadêmicos, a motivação para a obtenção de novos conhecimentos. Durante a realização do Quiz, cada grupo obtinha uma pontuação referente ao número de acertos. Essa pontuação permitia o acesso a recursos que posteriormente seriam utilizados no projeto do ciclone.

14 - Centrifugação é o processo físico de separação de misturas, onde a sedimentação, que ocorre de forma lenta, é acelerada por forças centrípetas. Para que isto ocorra, a mistura gira a uma grande velocidade, e o material mais denso, é depositado no fundo por inércia. Desse modo, quais os tipos de misturas que podem ser separadas por centrifugação?

a) Misturas sólido/líquido ou líquido/líquido, onde a força centrípeta faz com que o material mais denso se deposite no fundo do frasco ou saia pela tangente.

b) Misturas sólido/líquido ou líquido/líquido e sólido/sólido, pela diferença de densidade.

Misturas líquido/líquido, sólido/sólido e gás/gás, já que a força centrípeta só age em misturas que estejam no mesmo estado físico.

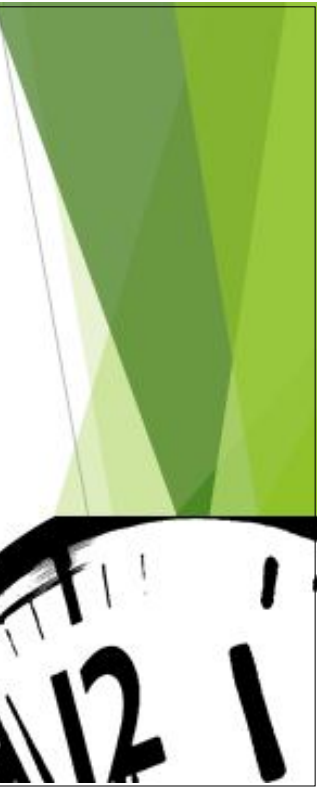

Figura 5 - Modelo de questão, utilizado durante a realização do Quiz

A ferramenta Quiz já foi utilizada em outras atividades realizadas pelo grupo $P E T-E Q$, e demonstrou ser uma excelente ferramenta de estratégia na promoção do aumento de interesse dos acadêmicos na busca pelo conhecimento de Engenharia. $O$ processo de aprendizagem nos níveis de graduação é dividido em etapas, para que os acadêmicos possam, gradativamente, ter condições de amadurecer conceitos básicos e assim ter condições de compreender conceitos mais avançados. Assim, do ponto de vista didático, o modelo pode ser configurado como válido, pois os acadêmicos visualizam a importância de desenvolver um conhecimento aprofundado e sólido. Entretanto, enxerga-se o 
desinteresse do aprendizado nos níveis iniciais e intermediários da graduação; a aplicação do Quiz revelou que, tal desinteresse surge, pois, há poucos momentos de conexão e interdisciplinaridade trabalhada sobre os conceitos envolvidos em sala de aula.

$\mathrm{Na}$ sequência, cada grupo recebeu um Cartão de Projeto, que continha os dados de projeto do ciclone que iria ser utilizado em um determinado setor da indústria. Para auxiliar os acadêmicos na construção do equipamento, foi disponibilizado um Cartão de Dicas (Figura 6), que continha as informações necessárias para o desenvolvimento do projeto. Além disso, para realizar a construção, negociação e visualização de todos os recursos utilizados no processo de fabricação e venda do equipamento, os grupos foram orientados a aplicar o Business Model Canvas. Desta forma, os participantes puderam projetar um ciclone em escala real e, com a utilização dos materiais, previamente adquiridos durante a realização do Quiz, construir um protótipo do equipamento.

\section{GARTÃo DE DICAS}

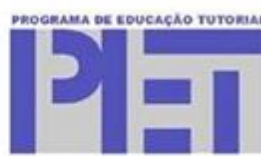

- O ciclone é um equipamento centrífugo utilizado para a separação de mistura. Suas dimensões e o material de que é fabricado pode variar de acordo com a especificidade da mistura a ser separada.

- As dimensões de um ciclone de Lapple podem ser encontradas através das seguintes relações:

$$
B=\frac{(D c p)^{2} 2 \pi N V\left(\rho_{s}-\rho_{f}\right)}{9 \mu} \quad L=Z=2 D c \quad D s=\frac{D c}{2} \quad J=\frac{D c}{2} \quad i=\frac{D c}{1,6} \quad B=\frac{D c}{4}
$$

Onde: $\boldsymbol{\mu}$ é viscosidade do fluido; $\mathbf{B}$ é largura do duto de entrada do ciclone; $\mathbf{V}$ é a velocidade do fluido que entra no ciclone; $\boldsymbol{\rho}_{\mathrm{f}}$ é a densidade do fluido, $\boldsymbol{\rho}_{\mathrm{s}}$ é a densidade da partícula sólida; $\mathbf{N}=\mathbf{5}$ e Dcp é o diâmetro de corte da partícula.

- O aço é um material versátil, pode ser utilizado para os mais diversos fins. Mas fique atento para o custo-benefício.

- O planejamento é a melhor maneira de se chegar a resultados rápidos e eficientes.

- A densidade e viscosidade dos principais fluidos utilizados nesse tipo de equipamento, a $20^{\circ} \mathrm{C}$ e $1 \mathrm{~atm}$, são:

\begin{tabular}{|l|l|l|}
\hline Fluido & $\begin{array}{l}\text { Viscosidade } \\
(\mathrm{Pa} . \mathrm{s})\end{array}$ & Densidade $\left(\mathrm{kg} / \mathrm{m}^{3}\right)$ \\
\hline $\mathrm{Ar}$ & $1,81 * 10^{-5}$ & 1,204 \\
\hline Água & 1,005 & 998,2 \\
\hline
\end{tabular}

Fonte: GEANKOPLIS, C. J. Transport process and unit operations. 3ed. Englewood Cliffs: PrenticeHall, 1998.

Figura 6 - Modelo de Cartão de Dicas, utilizado no desenvolvimento da atividade

A utilização do BMC permitiu que os estudantes enxergassem as etapas envolvidas na produção de um equipamento industrial aplicado na área de Engenharia, contabilizassem as variáveis mercadológicas que influenciam na estruturação do projeto e, ainda, desenvolvessem seus produtos atentando para as necessidades de seus clientes, com foco na otimização no uso de recursos e logística. A utilização da ferramenta de gestão proporcionou aos alunos, também, simular um estudo sobre a viabilidade de implantação de um equipamento, no setor de uma indústria que trabalha com equipamentos de separação sólido-fluido. 
Todo o processo de ensino-aprendizagem demonstrou ser bastante produtivo e eficaz. A aplicação da atividade com a participação dos quatorze acadêmicos propiciou visualizar $\mathrm{o}$ maior envolvimento $\mathrm{e}$ desenvoltura de cada participante. Ao se utilizar o formato proposto como uma ferramenta de indissociabilidade dos conteúdos abordados em sala de aula, em conjunto com a aplicação do BMC para o auxílio no desenvolvimento e contextualização do projeto do ciclone, foi possível perceber que os acadêmicos se sentiram mais motivados e todo o trabalho se tornou mais próximo daquele realizado na prática profissional do Engenheiro. Por fim, mediante a análise das respostas do Questionário Avaliativo de Atividade, o Grupo PET EQ constatou que a averiguação dos resultados, supracitados, foi corretamente realizada, pois corroborou com a avaliação apresentada pelos participantes no QAA.

Dessa forma, presume-se que, ao inserir mais variáveis à equação e transfigurar o processo mais dependente de fatores externos, foi possível ampliar a visão macro de Engenharia que o graduando necessita desenvolver durante o curso. Faz-se necessário, ainda, visualizar a realidade do acadêmico, e auxiliá-lo no intuito de possibilitar a sua atuação como sujeito ativo na construção de seu conhecimento, e não como agente passivo, ao ser apenas ouvinte durante $o$ processo do ensinoaprendizagem.

O estudante deverá ser induzido a desenvolver um método natural razoável para adquirir um grau de autoconfiança e firmeza na abordagem de novos problemas, o que será de inestimável valor na sua prática em engenharia. $O$ estudante deve também ser treinado para defender seus pontos de vista e para demonstrar, de forma concisa e clara, a importância e as finalidades requeridas de todos os exercícios que currículo possa oferecer (OLIVEIRA; PINTO, 2006).

Observa-se, dessa forma, que é imprescindível que os conhecimentos sejam colocados em prática e que os profissionais das áreas de Engenharia estejam atentos a seus papéis na sociedade como conhecedores dos próprios ramos de atuação. Silva e Cecílio (2007) corroboram com tal afirmação, e alegam, sobre os estudantes de engenharia:

Precisam aprender a fazer, a fim de adquirir não somente uma qualificação profissional, mas, de uma maneira mais ampla, competências que tornem a pessoa apta a enfrentar numerosas situações e a trabalhar em equipe. (SILVA CECILIO, 2007)

Ao considerar, também, a necessidade de se proporcionar mais dinamismo na formação de profissionais e criar espaços de maior motivação para o aprendizado de Engenharia, diferentes autores utilizam um conceito denominado "reencantamento da educação"; este conceito tomou forma através das publicações do teólogo brasileiro Hugo Assmann.

\begin{abstract}
Reencantar a educação significa vivenciar a vida aprendendo e aprender rumo a uma sociedade aprendente, com todas as suas implicações pedagógicas - do plano biofísico às esferas societais -, ou seja, a educação deve abranger o sentido de todas as formas de vida, pois os processos cognitivos e os vitais são, em síntese, a mesma coisa (ASSMANN, 1998, apud CAMOLEZ; JARDILINO, 2005, p. 112).
\end{abstract}

Observa-se que, quanto ao ensino de Engenharia Química, os processos e equipamentos não realizam suas funções isoladamente e toda a cadeia de produção e consumo é interligada e funciona como um grande organismo vivo.

$\mathrm{Na}$ realidade, nos esquecemos que todo fato histórico ocorre num espaço geográfico, que os aprendizes estão inseridos em contextos significativos e que sentimento, emoção e razão envolvem processos interdependentes e inseparáveis em nossa corporeidade (MORAES, 2004).

\section{CONCLUSÃo}

Ao contextualizar o acadêmico na estruturação de um projeto de equipamento utilizado na indústria de Engenharia Química, mediante o desenvolvimento da Atividade Integralizadora, revelou-se a necessidade de adaptar e inserir novas formas de aprendizado no ensino de Engenharia. A criação de ferramentas 
dinâmicas, com caráter instigador, tornam os estudantes mais participativos no processo de construção do saber e possibilitam a formação de profissionais de excelência, aptos a enfrentar as realidades do mercado atual.

\section{REFERÊNCIAS}

BUSINESS MODEL GENERATION. Business Model Canvas. Zuerich: Business Model Foundry, 2014. Disponível em http://www.businessmodelgeneration.co m/. Acesso em: 18 Abr. 2014.

CAMOLEZ, J.C.; JARDILINO, J.R.L. Reencantar a educação é possível? Cadernos de Pós Graduação, São Paulo, v. 4, Educação, p. 111-120, 2005. Disponível em http://www.uninove.br/PDFs/Publicac oes/cadernos posgraduacao/cadernosv4edu/cdposv4 n1edu2c10.pdf. Acesso em: 18 abr. 2014.

GEANKOPLIS, C.J. Transport Processes and Unit Operations. Third Edition. Ed. Prentice Hall P T R, Englewood Cliffs, New Jersey, 1993. 921 p.

MORAES, M.C. Reencantamento da educação a partir de novos paradigmas da ciência. Pontifícia Universidade Católica, São Paulo, Brasil, 2004. Disponível em http://www.ub.edu/sentipensar/pdf/cand ida/reencantar educacao.pdf. Acesso em: 18 abr. 2014.

OLIVEIRA, V.F.; PINTO, D.P. Educação em Engenharia como área do conhecimento. Anais do XXXIV COBENGE, Passo Fundo: Ed. Universidade de Passo Fundo, 2006. Disponível em http://www.dee.uf ma.br/ fsouza/anais/arquivos/12 85 357.pdf. Acesso em: 18 abr. 2014.

RIEDER, R.; ZANELATTO, E.M.; BRANCHER, J.D. Observação e Análise da Aplicação de Jogos Educacionais Bidimensionais Em um Ambiente Aberto. IX Taller Internacional de Software Educativo TISE 2004. Universidade Regional Integrada do Alto Uruguai e das Missões - Campus de Erexim, Brasil. Journal of Computer Science, v.4, n.2, 2005. Disponível em http://www.dcc.ufla.br/infocomp/artigos/ v4.2/art08.pdf Acesso em: 18 abr. 2014.
SILVA, J. M. de A.; CANEDO, R.V.; ABRANTES, T.A. de S.; SANTOS, R.T. dos; SOUZA, R.A.; UTAGAWA, C.Y. Quiz: um Questionário Eletrônico para Autoavaliação e Aprendizagem em Genética e Biologia Molecular. Revista Brasileira de Educação Médica, v.34, n.4, 2010. Disponível em http://www.scie lo.br/pdf/rbem/v34n4/v34n4a17.pdf. Acesso em: 18 abr. 2014.

SILVA, L.P.; CECÍLIO, S. A mudança no modelo de ensino e de formação na engenharia. Educação em Revista, Belo Horizonte, v. 45, p. 61-80, 2007. Disponível em http://www.scielo.br/scielo.php?script=s ci arttext\&pid=S0102-46982007000100004. Acesso em: 18 abr. 2014.

SILVA, M.K. Estudo de Modelagem Numérica Tridimensional de Ciclones do Tipo Lapple para a Separação Gás-Sólido. 2006. 79 f. Dissertação submetida ao Programa de Pós-Graduação em Engenharia Química do Centro Tecnológico da Universidade Federal de Santa Catarina, Florianópolis, 2006. Disponível em https://repositorio.ufsc.br/bitstrea $\mathrm{m} /$ handle/123456789/89536/226538.pdf? sequence $=1$. Acesso em: 18 abr. 2014.

VASCONCELOS, R.; ALMEIDA, L.S.; MONTEIRO, S. 0 insucesso e abandono acadêmico na universidade: uma análise sobre os cursos de engenharia. Anais do VI International Conference on Engineering and Computer Education. Buenos Aires, Argentina. 2009. Disponível em http://repositorium.sdu m.uminho.pt/handle/1822/9412. Acesso em: 18 Abr. 2014. 